\title{
On the geometric realization and subdivisions of dihedral sets
}

\author{
SHO SAITO
}

\begin{abstract}
We extend to dihedral sets Drinfeld's filtered-colimit expressions of the geometric realization of simplicial and cyclic sets. We prove that the group of homeomorphisms of the circle continuously act on the geometric realization of a dihedral set. We also see how these expressions of geometric realization clarify subdivision operations on simplicial, cyclic and dihedral sets defined by Bökstedt, Hsiang and Madsen, and Spaliński.
\end{abstract}

18G30; 55U10

\section{Introduction}

By expressing the geometric realization of simplicial sets and cyclic sets as filtered colimits, Drinfeld [2] proved in a substantially simplified way the fundamental facts that geometric realization preserves finite limits, and that the group of orientation-preserving homeomorphisms of the interval $[0,1]$ (resp. the circle $\mathbb{R} / \mathbb{Z}$ ) acts on the realization of a simplicial (resp. cyclic) set. In this paper, we extend his result to dihedral sets. We also see how these expressions lead to a clarified description of subdivision operations, which were introduced by Bökstedt, Hsiang and Madsen [1] for simplicial and cyclic sets, and by Spaliński [6] for dihedral sets. In Spaliński's definition, the subdivisions of dihedral sets are merely simplicial sets. We prove that they in fact have richer structures. We have strived to make the exposition self-contained and to give complete proofs of the stated results.

\subsection{Terminology and results}

Firstly, we review here the results of Drinfeld [2] on geometric realization. Let $\Delta$ be the simplicial index category of the finite linearly ordered sets $[n]=\{0<\cdots<n\}, n \geq 0$, and order-preserving maps. By definition, a simplicial set is a contravariant functor $X[\cdot]$ from $\Delta$ to the category Sets of sets. We write $\Delta[n][\cdot]$ for the standard simplicial $n$-simplex $\operatorname{Hom}_{\Delta}([\cdot],[n])$ and set $\Delta[n]=\left\{\left(z_{0}, \ldots, z_{n}\right) \in[0,1]^{n+1} \mid \sum_{i=0}^{n} z_{i}=1\right\}$, with the standard Euclidean topology. 
Definition 1.1 (Milnor [4]) The geometric realization $|X[\cdot]|$ of the simplicial set $X[\cdot]$ is the colimit

$$
\operatorname{colim}_{\Delta[n][\cdot] \rightarrow X[\cdot]} \Delta[n],
$$

where the index category is formed by simplicial maps from standard simplicial simplices to $X[\cdot]$ and natural transformations.

We can construct $|X[\cdot]|$ explicitly by

$$
|X[\cdot]|=\coprod_{n \geq 0} X[n] \times \Delta[n] / \sim,
$$

where $X[n]$ is given the discrete topology and where $\sim$ is the equivalence relation generated by the relation that identifies $\left(x, \theta_{*} z\right)$ with $\left(\theta^{*} x, z\right)$ for every pair $(x, z) \in$ $X[n] \times \Delta[m]$ and for every map $\theta:[m] \rightarrow[n]$ in $\Delta$. However, we interchangeably adopt any other space having the universal property of the colimit as a definition of $|X[\cdot]|$, since there are canonical isomorphisms between such spaces.

Drinfeld [2] re-defined the geometric realization as a filtered colimit, showing that his definition is equivalent to Milnor's. To introduce his expression, we first need to extend the simplicial set $X[\cdot]$ to a contravariant functor $\tilde{X}[\cdot]$ from the category $\Delta_{\text {big }}$ of all non-empty finite linearly ordered sets. We denote by $\mathcal{F}$ the set of all finite subsets of $[0,1]$, viewed as a category with morphisms being inclusions. For each $F \in \mathcal{F}$, we order the set of connected components $\pi_{0}([0,1] \backslash F)$ by declaring that $[x] \leq[y]$ if $x \leq y \in[0,1] \backslash F$. If $F \subset G$ there is an order-preserving map $\pi_{0}([0,1] \backslash G) \rightarrow \pi_{0}([0,1] \backslash F)$.

Theorem 1.1 (Drinfeld [2]) The geometric realization $|X[\cdot]|$ of the simplicial set $X[\cdot]$ is given by the colimit

$$
\operatorname{colim}_{F \in \mathcal{F}} \tilde{X}\left[\pi_{0}([0,1] \backslash F)\right] .
$$

The point is that the index category $\mathcal{F}$ is filtering, so it results as an immediate consequence that geometric realization, at least as a functor from the category of simplicial sets to Sets, preserves finite limits. This expression also makes it obvious that the group $\operatorname{Homeo}([0,1], \partial[0,1])$ of order-preserving homeomorphisms of $[0,1]$ acts on the set $|X[\cdot]|$.

In fact, these statements still hold when the geometric realization is considered to take values in the category of $k$-spaces. Drinfeld [2] proved that the topology on $|X[\cdot]|$ given in Definition 1.1 can be described by the metric $d$ on the above colimit, defined as follows. For every $F \in \mathcal{F}$, we write $\mu_{F}$ for the measure on $\pi_{0}([0,1] \backslash F)$ 
defined by $\mu_{F}(A)=\sum_{c \in A} l(c)$. Here $A$ is a subset of $\pi_{0}([0,1] \backslash F)$ and $l(c)$ denotes the length of the path-connected component of $[0,1] \backslash F$ corresponding to $c \in A$, ie, if $c$ is the interval between $x \in F$ and $y \in F$ then $l(c)=|x-y|$. Take two elements of $\operatorname{colim}_{F \in \mathcal{F}} \tilde{X}\left[\pi_{0}([0,1] \backslash F)\right]$. Since $\mathcal{F}$ is filtering, we may assume these to be represented by elements $u$ and $v$ of $\tilde{X}\left[\pi_{0}([0,1] \backslash F)\right]$ with some common $F \in \mathcal{F}$. We define the distance of the two elements to be the minimum of $\mu_{F}\left(\pi_{0}([0,1] \backslash F) \backslash A\right)$ with respect to subsets $A$ of $\pi_{0}([0,1] \backslash F)$ such that the map $\tilde{X}\left[\pi_{0}([0,1] \backslash F)\right] \rightarrow \tilde{X}[A]$ takes $u$ and $v$ to an identical element. (If there does not exist such an $A$, we set the distance to be 1.) See [2] for details.

A similar argument applies to cyclic sets introduced by Connes. Let $\Delta C$ be the category that makes the family $\left\{C_{n+1}\right\}_{n \geq 0}$ of cyclic groups of order $n+1$ into a crossed simplicial group in the sense of Fiedorowicz and Loday [3]. Recall:

Definition 1.2 (Fiedorowicz and Loday [3]) A crossed simplicial group is a family of groups $\left\{G_{n}\right\}_{n \geq 0}$ together with a category $\Delta G$ that has one object $[n]$ for each $n \geq 0$, containing $\Delta$ as a subcategory, and satisfies the following conditions:

(1) The group of automorphisms Aut $_{\Delta G}[n]$ on each $[n]$ is isomorphic to the group $G_{n}^{\mathrm{op}}$.

(2) Every morphism $[m] \rightarrow[n]$ can be uniquely written as a composite $\phi \circ g$ with $\phi \in \operatorname{Hom}_{\Delta}([m],[n])$ and $g \in \operatorname{Aut}_{\Delta G}[m]$.

For a $\Delta G$-set $X[\cdot]$ (ie, a contravariant functor from $\Delta G$ to Sets), we define its geometric realization to be the realization of the underlying simplicial set

$$
\Delta^{\mathrm{op}} \hookrightarrow(\Delta G)^{\mathrm{op}} \stackrel{X[\cdot]}{\longrightarrow} \text { Sets . }
$$

Cyclic sets are defined to be $\Delta C$-sets. Drinfeld [2] constructed $\Delta C$ as the category of the $\mathbb{Z}_{+}$-categories $[n]_{\text {cyc }}, n \geq 0$, and $\mathbb{Z}_{+}$-functors, as recalled in Section 2 below.

The cyclic set $X[\cdot]:(\Delta C)^{\text {op }} \rightarrow$ Sets can be extended to a contravariant functor $\tilde{X}[\cdot]$ from the extended category $\Delta_{\text {big }} C$ of $\mathbb{Z}_{+}$-categories isomorphic to some $[n]_{\text {cyc }}$. Write $\mathcal{F}^{\prime}$ for the set of all finite subsets of $\mathbb{R} / \mathbb{Z}$, viewed as a filtered category.

Theorem 1.2 (Drinfeld [2]) The geometric realization $|X[\cdot]|$ of the cyclic set $X[\cdot]$ is given by the filtered colimit

$$
\operatorname{colim}_{F \in \mathcal{F}^{\prime}} \tilde{X}\left[\pi_{0}(\mathbb{R} / \mathbb{Z} \backslash F)\right] .
$$

In particular, the group Homeo ${ }^{+} \mathbb{R} / \mathbb{Z}$ of orientation-preserving homeomorphisms of the circle $\mathbb{R} / \mathbb{Z}$ acts continuously on $|X[\cdot]|$. 
Our result on the realization of dihedral sets is analogous to the above theorem. A dihedral set $X[\cdot]$ is a $\Delta D$-set, where $\Delta D$ is the category that makes the family $\left\{D_{n+1}\right\}_{n \geq 0}$ of the dihedral groups of order $2(n+1)$ into a crossed simplicial group (Fiedorowicz and Loday [3]). Fiedorowicz and Loday [3] constructed $\Delta D$ by giving a presentation of it, ie, by specifying generators and relations. We show that $\Delta D$ can be equivalently introduced as the category of $[n]_{\text {cyc }}, n \geq 0$, and covariant and contravariant $\mathbb{Z}_{+}$-functors.

There is a similar extension of the dihedral set $X[\cdot]:(\Delta D)^{\mathrm{op}} \rightarrow$ Sets to a contravariant functor $\tilde{X}[\cdot]$ from the extended category $\Delta_{\text {big }} D$ of $\mathbb{Z}_{+}-$categories isomorphic to some $[n]_{\text {cyc }}$ and covariant and contravariant $\mathbb{Z}_{+}-$functors. It is known that the geometric realization of a dihedral set admits a continuous action by the orthogonal group $O(2)$. We prove the following new, stronger result:

Theorem 1.3 The geometric realization $|X[\cdot]|$ of the dihedral set $X[\cdot]$ is given by the filtered colimit

$$
\operatorname{colim}_{F \in \mathcal{F}} \tilde{X}\left[\pi_{0}(\mathbb{R} / \mathbb{Z} \backslash F)\right] .
$$

In particular, the group Homeo $\mathbb{R} / \mathbb{Z}$ of all homeomorphisms of the circle $\mathbb{R} / \mathbb{Z}$ acts continuously on $|X[\cdot]|$.

From these filtered-colimit expressions, we find a clearer description of subdivision operations. For every positive integer $r$, Bökstedt, Hsiang and Madsen [1] defined an operation called the $r$-fold edgewise subdivision of simplicial or cyclic sets. We denote by $\operatorname{sd}_{r} X[\cdot]$ the $r$-fold edgewise subdivision of the simplicial or cyclic set $X[\cdot]$, whose definition is recalled in Section 3. Write $\mathcal{F}_{r}$ and $\mathcal{F}_{r}^{\prime}$ for the set of finite subsets of $[0, r]$ and $\mathbb{R} / r \mathbb{Z}$, respectively. We introduce the following expression for subdivisions, which gives a new natural proof of the result by Bökstedt, Hsiang and Madsen [1] that $|X[\cdot]|$ and $\left|\operatorname{sd}_{r} X[\cdot]\right|$ are canonically homeomorphic.

Theorem 1.4 For the simplicial (resp. cyclic) set $X[\cdot]$, the realization $\left|\operatorname{sd}_{r} X[\cdot]\right|$ is given by the filtered colimit

$$
\operatorname{colim}_{F \in \mathcal{F}_{r}} \tilde{X}\left[\pi_{0}([0, r] \backslash F)\right] \quad\left(\text { resp. } \operatorname{colim}_{F \in \mathcal{F}_{r}^{\prime}} \tilde{X}\left[\pi_{0}(\mathbb{R} / r \mathbb{Z} \backslash F)\right]\right)
$$

and hence admits an action by

$$
G_{r}=\operatorname{Homeo}([0, r], \partial[0, r]) \quad\left(\text { resp. } G_{r}=\text { Homeo }^{+} \mathbb{R} / r \mathbb{Z}\right) .
$$

In particular, the bijection $[0, r] \rightarrow[0,1]$ (resp. $\mathbb{R} / r \mathbb{Z} \rightarrow \mathbb{R} / \mathbb{Z}$ ) given by $x \mapsto x / r$ induces isomorphisms $D_{r}:\left|\operatorname{sd}_{r} X[\cdot]\right| \rightarrow|X[\cdot]|$ and $d_{r}: G_{r} \rightarrow G=\operatorname{Homeo}([0,1], \partial[0,1])$ 
(resp. $d_{r}: G_{r} \rightarrow G=$ Homeo $^{+} \mathbb{R} / r \mathbb{Z}$ ) such that the diagram

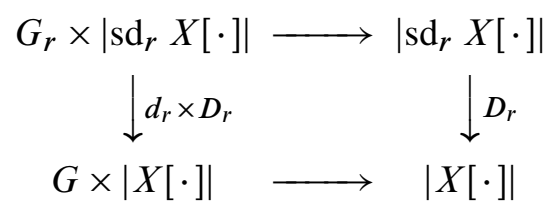

commutes.

Bökstedt, Hsiang and Madsen [1] used the diagonal embedding $\Delta[n] \rightarrow \Delta[r(n+1)-1]$, $u \mapsto(u / r, \ldots, u / r)$, to give a homeomorphism $\left|\operatorname{sd}_{r} X[\cdot]\right| \rightarrow|X[\cdot]|$, but it required some effort to construct its inverse and to prove that the homeomorphism is compatible with the appropriate actions on both sides. In contrast, our homeomorphism induced from $x \mapsto x / r$ is clear to have an inverse and to make the diagram above commute. (The same thing can be said to Theorem 1.5 below.)

For dihedral sets, Spaliński [6] defined two types of subdivision operations, $\operatorname{sd}_{r}$ and $\operatorname{sd}_{r}^{\mathrm{e}}$. In his definition, both operations assign to a dihedral set $X[\cdot]$ a simplicial set. We re-define in Section $3 \operatorname{sd}_{r} X[\cdot]$ and $\operatorname{sd}_{r}^{\mathrm{e}} X[\cdot]$ to have the richer structures of a $\Delta_{r} D$-set and a $\Delta_{2 r} D$-set, respectively. Here $\Delta_{r} D$ is the category that makes $\left\{D_{r(n+1)}\right\}_{n \geq 0}$ into a crossed simplicial group.

Theorem 1.5 For the dihedral set $X[\cdot],\left|\mathrm{sd}_{r} X[\cdot]\right|\left(\operatorname{resp} .\left|\mathrm{sd}_{r}^{\mathrm{e}} X[\cdot]\right|\right)$ is given by the filtered colimit

$$
\operatorname{colim}_{F \in \mathcal{F}_{r}^{\prime}} \tilde{X}\left[\pi_{0}(\mathbb{R} / r \mathbb{Z} \backslash F)\right] \quad\left(\text { resp. } \operatorname{colim}_{F \in \mathcal{F}_{2 r}} \tilde{X}\left[\pi_{0}(\mathbb{R} / 2 r \mathbb{Z} \backslash F)\right]\right)
$$

and hence admits an action by $G_{r}=$ Homeo $\mathbb{R} / r \mathbb{Z}$ (resp. $G_{r}^{\mathrm{e}}=$ Homeo $\left.\mathbb{R} / 2 r \mathbb{Z}\right)$. In particular, the bijection $\mathbb{R} / r \mathbb{Z} \rightarrow \mathbb{R} / \mathbb{Z}$ (resp. $\mathbb{R} / 2 r \mathbb{Z} \rightarrow \mathbb{R} / \mathbb{Z}$ ) given by $x \mapsto x / r$ (resp. $x \mapsto x /(2 r))$ induces isomorphisms $D_{r}:\left|\operatorname{sd}_{r} X[\cdot]\right| \rightarrow|X[\cdot]|\left(\right.$ resp. $D_{r}^{\mathrm{e}}:\left|\operatorname{sd}_{r}^{\mathrm{e}} X[\cdot]\right| \rightarrow$ $|X[\cdot]|)$ and $d_{r}: G_{r} \rightarrow G=$ Homeo $\mathbb{R} / \mathbb{Z}\left(\operatorname{resp} . d_{r}^{\mathrm{e}}: G_{r}^{\mathrm{e}} \rightarrow G=\right.$ Homeo $\left.\mathbb{R} / \mathbb{Z}\right)$ such that the diagram

$$
\begin{aligned}
& G_{r} \times\left|\operatorname{sd}_{r} X[\cdot]\right| \longrightarrow\left|\operatorname{sd}_{r} X[\cdot]\right| \quad G_{r} \times\left|\operatorname{sd}_{r}^{\mathrm{e}} X[\cdot]\right| \longrightarrow\left|\mathrm{sd}_{r}^{\mathrm{e}} X[\cdot]\right| \\
& \downarrow^{D_{r} \times d_{r}} \quad \downarrow_{r} \quad \text { resp. } \quad D_{r} \times D_{r}^{\mathrm{e}} \quad \downarrow_{r}^{\mathrm{e}} \\
& G \times|X[\cdot]| \longrightarrow|X[\cdot]| \quad G \times|X[\cdot]| \longrightarrow|X[\cdot]|
\end{aligned}
$$

commutes.

Finally, we will explain how $\operatorname{sd}_{r}^{\mathrm{e}} X[\cdot]$ admits simplicial actions by $D_{r}$ and $C_{r}$, and hence defines simplicial sets $\left(\mathrm{sd}_{r}^{\mathrm{e}} X[\cdot]\right)^{D_{r}}$ and $\left(\mathrm{sd}_{r}^{\mathrm{e}} X[\cdot]\right)^{C_{r}}$, respectively, and see that the latter one again has the structure of a dihedral set. 
Acknowledgements I would like to thank Lars Hesselholt for his valuable comments and discussions. I am also grateful to Jean-Louis Loday for making a correction to the references.

\section{The proof of Theorem 1.3}

\subsection{Geometric realization of dihedral sets}

We first recall Drinfeld's construction of $\Delta C$ as a category of $\mathbb{Z}_{+}-$categories [2]. Here $\mathbb{Z}_{+}$is the additive monoid of non-negative integers, and a $\mathbb{Z}_{+}-$category is a category $\mathcal{C}$ together with a nontrivial monoid map $\mathbb{Z}_{+} \rightarrow \operatorname{End}_{\mathcal{C}} \mathrm{id}_{\mathcal{C}}$. Ie, there is a non-identity endomorphism $1_{c}: c \rightarrow c$ on every object $c \in$ ob $\mathcal{C}$ such that $f \circ 1_{c_{1}}=1_{c_{2}} \circ f$ for every $f: c_{1} \rightarrow c_{2}$. A $\mathbb{Z}_{+}-$functor is a functor between $\mathbb{Z}_{+}$-categories that preserves the structural endomorphisms.

The most basic example of a $\mathbb{Z}_{+}$-category is the circle $\mathbb{R} / \mathbb{Z}$. Morphisms from $x$ to $y$ are homotopy classes of continuous maps $f:[0,1] \rightarrow \mathbb{R} \rightarrow \mathbb{R} / \mathbb{Z}$ such that $f(0)=x$ and $f(1)=y$, with $[0,1] \rightarrow \mathbb{R}$ non-decreasing and $\mathbb{R} \rightarrow \mathbb{R} / \mathbb{Z}$ the canonical projection. The $\mathbb{Z}_{+}$-category structure is given by $1_{x}=($ class of degree 1 loops based at $x$ ). If $F \subset \mathbb{R} / \mathbb{Z}$ is a finite subset, the set of connected components $\pi_{0}(\mathbb{R} / \mathbb{Z} \backslash F)$ can be considered as a $\mathbb{Z}_{+}$-category. The set of morphisms from $c$ to $d$ is defined by choosing representatives $x_{c} \in c$ and $x_{d} \in d$ :

$$
\operatorname{Hom}_{\pi_{0}(\mathbb{R} / \mathbb{Z} \backslash F)}(c, d)=\operatorname{Hom}_{\mathbb{R} / \mathbb{Z}}\left(x_{c}, x_{d}\right)
$$

If $F \subset G$ there is a $\mathbb{Z}_{+}$-functor $\pi_{0}(\mathbb{R} / \mathbb{Z} \backslash G) \rightarrow \pi_{0}(\mathbb{R} / \mathbb{Z} \backslash F)$.

There is a way of constructing a $\mathbb{Z}_{+}$-category $\mathcal{A}_{\text {cyc }}$ from a given small category $\mathcal{A}$ [2, Example 4 of Section 2]. In the particular case where $\mathcal{A}=\left\{a_{0}<\cdots<a_{n}\right\}$ is a linearly ordered set, viewed as a category, then $\mathcal{A}_{\text {cyc }}$ is the $\mathbb{Z}_{+}$-category that has the same objects as $\mathcal{A}$ and that has morphisms generated by those in $\mathcal{A}$ together with one new generator $a_{n} \rightarrow a_{0}$. The structural endomorphisms are given by $1_{a_{i}}: a_{i} \rightarrow a_{n} \rightarrow$ $a_{0} \rightarrow a_{i}$. If $\mathcal{A}=[n]$ then $[n]_{\text {cyc }}$ is identified with the full $\mathbb{Z}_{+}$-subcategory

$$
\{[0],[1 /(n+1)], \ldots,[n /(n+1)]\} \subset \mathbb{R} / \mathbb{Z},
$$

where $[\cdot]$ denotes the class in $\mathbb{R} / \mathbb{Z}$. (For notational simplicity we will frequently omit such brackets.) The cyclic index category $\Delta C$ (resp. $\Delta_{\text {big }} C$ ) is defined to be the category of the $\mathbb{Z}_{+}$-categories $[n]_{\text {cyc }}$ (resp. small $\mathbb{Z}_{+}$-categories isomorphic to some $\left.[n]_{\text {cyc }}\right)$ and $\mathbb{Z}_{+}-$functors. 
Analogously, we set $\Delta D$ to be the category whose set of objects is the same as that of $\Delta C$, and whose set of morphisms from $[m]_{\text {cyc }}$ to $[n]_{\text {cyc }}$ is the disjoint union of the sets of covariant and contravariant $\mathbb{Z}_{+}$-functors from $[m]_{\text {cyc }}$ to $[n]_{\text {cyc }}$.

Remarks (1) If $C$ is a $\mathbb{Z}_{+}$-category, the $\mathbb{Z}_{+}$-category structure on the opposite category $C^{\mathrm{op}}$ is given by $1_{c}=\left(1_{c}\right)^{\mathrm{op}}$ for $c \in \mathrm{ob} C^{\mathrm{op}}$. (2) Considering the disjoint union means that a functor cannot be both covariant and contravariant. As a consequence, eg, $\operatorname{Hom}_{\Delta D}\left([0]_{\text {cyc }},[0]_{\text {cyc }}\right)$ has two elements: the identity $\mathrm{id}_{[0]_{\text {cyc }}}:[0]_{\text {cyc }} \rightarrow[0]_{\text {cyc }}$ as a covariant functor, and $\mathrm{id}_{[0]_{\mathrm{cyc}}}:[0]_{\mathrm{cyc}} \rightarrow[0]_{\mathrm{cyc}}^{\mathrm{op}}$ as a contravariant functor.

The composition in $\Delta D$ is defined by usual composition of functors, under the rule that the composite of two covariant or contravariant functors should be covariant, and the composite of covariant and contravariant functors should be contravariant.

The following proposition shows that $\Delta D$ is the correct dihedral index category.

Proposition 2.1 The category $\Delta D$ makes the family $\left\{D_{n+1}\right\}_{n \geq 0}$ into a crossed simplicial group.

Proof We have to check Conditions (1) and (2) of Definition 1.2.

(1) For each $n \geq 0$, define $\mathbb{Z}_{+}$-functors

$$
\tau_{n}:[n]_{\mathrm{cyc}} \rightarrow[n]_{\mathrm{cyc}} \quad \text { and } \quad \omega_{n}:[n]_{\mathrm{cyc}} \rightarrow[n]_{\mathrm{cyc}}^{\mathrm{op}}
$$

by $\tau_{n}(x)=1 /(n+1)+x$ and $\omega_{n}(x)=-1 /(n+1)-x$, respectively. Then $\tau_{n}$ and $\omega_{n}$ are isomorphisms in $\Delta D$ on $[n]_{\text {cyc }}$ of order $n+1$ and 2 , respectively, and satisfy the relation $\tau_{n} \omega_{n}=\omega_{n} \tau_{n}^{-1}$. In addition, any $\phi \in \operatorname{Aut}_{\Delta D}[n]_{\text {cyc }}$ can be written as a product of $\tau_{n}$ and $\omega_{n}$. Indeed, if $\phi$ is covariant, then $\phi \in \operatorname{Aut}_{\Delta C}[n]_{\text {cyc }}=\left\langle\tau_{n}\right\rangle$ is a power of $\tau_{n}$. If $\phi$ is contravariant, then $\phi \circ \omega_{n}$ is covariant, and so a power of $\tau_{n}$. Hence Aut $_{\Delta D}[n]_{\text {cyc }}$ is generated by $\tau_{n}$ and $\omega_{n}$. This means that

$$
\text { Aut }_{\Delta D}[n]_{\mathrm{cyc}}=\left\langle\tau_{n}, \omega_{n} \mid \tau_{n}^{n+1}=\omega_{n}^{2}=1, \tau_{n} \omega_{n}=\omega_{n} \tau_{n}^{-1}\right\rangle
$$

is the dihedral group of order $2(n+1)$.

(2) Let $\phi:[m]_{\text {cyc }} \rightarrow[n]_{\text {cyc }}$ be a map in $\Delta D$. If $\phi$ is a covariant functor, then $\phi \in$ $\operatorname{Hom}_{\Delta C}\left([m]_{\text {cyc }},[n]_{\text {cyc }}\right)$, so that it can be uniquely written as $\phi=\psi \circ g$ with $\psi$ being a map in $\Delta$ and $g \in \operatorname{Aut}_{\Delta C}[m]_{\text {cyc }} \subset$ Aut $_{\Delta D}[m]_{\text {cyc }}$. If $\phi$ is contravariant, then we can uniquely write $\phi \circ \omega_{m} \in \operatorname{Hom}_{\Delta C}\left([m]_{\text {cyc }},[n]_{\text {cyc }}\right)$ as a composite $\phi \circ \omega_{m}=\psi \circ g$ with $\psi$ in $\Delta$ and $g \in$ Aut $_{\Delta C}[m]_{\text {cyc }}$. Multiplication by $\omega_{m}$ on the right yields $\phi=\psi \circ g \circ \omega_{m}$ with $g \circ \omega_{m} \in$ Aut $_{\Delta D}[m]_{\text {cyc }}$. 
We extend the dihedral set $X[\cdot]$ to a functor $\tilde{X}[\cdot]:\left(\Delta_{\text {big }} D\right)^{\text {op }} \rightarrow$ Sets, by defining on objects $\tilde{X}[\lambda]=X\left[m_{\lambda}\right]_{\text {cyc }}$ and on morphisms $\tilde{X}[f]=X[\tilde{f}]: X\left[m_{\mu}\right]_{\text {cyc }} \rightarrow X\left[m_{\lambda}\right]_{\text {cyc }}$, where $f: \lambda \rightarrow \mu$ is a map in $\Delta_{\text {big }} D$ and $\tilde{f}$ is the unique map in $\Delta D$ that makes the following diagram commute:

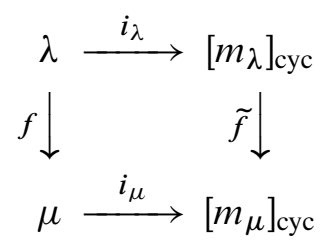

Equivalently, $\tilde{X}[\cdot]$ is the unique (up to unique isomorphism) map that is identical to $X[\cdot]$ on $\Delta D^{\text {op }}$. For example, if $X[\cdot]$ is the standard dihedral set $\Lambda_{\operatorname{dih}}[n][\cdot]=$ $\operatorname{Hom}_{\Delta D}\left([\cdot],[n]_{\text {cyc }}\right)$, the extension $\widehat{\Lambda_{\text {dih }}[n]}[\cdot]$ is given by $\lambda \mapsto \operatorname{Hom}_{\Delta_{\text {big }} D}\left(\lambda,[n]_{\text {cyc }}\right)$.

By definition, $|X[\cdot]|$ is the realization of the underlying cyclic set $\left.X\right|_{(\Delta C)^{\text {op }}}[\cdot]$. Hence we have

$$
|X[\cdot]|=|X|_{(\Delta C)^{\text {op }}}[\cdot] \mid=\operatorname{colim}_{F \in \mathcal{F}^{\prime}} \overline{\left(\left.X\right|_{(\Delta C)^{\text {op }}}\right)}\left[\pi_{0}(\mathbb{R} / \mathbb{Z} \backslash F)\right] .
$$

The extension $\overline{\left(\left.X\right|_{(\Delta C)^{\text {op }}}\right)}[\cdot]$ of $\left.X\right|_{(\Delta C)^{\text {op }}[\cdot]}$ to $\left(\Delta_{\text {big }} C\right)^{\text {op }}$ is nothing but the restriction

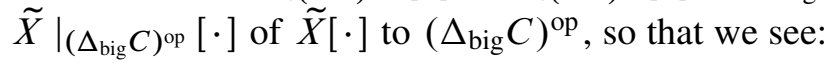

$$
|X[\cdot]|=\left.\operatorname{colim}_{F \in \mathcal{F}^{\prime}} \tilde{X}\right|_{\left(\Delta_{\text {big }} C\right)^{\text {op }}}\left[\pi_{0}(\mathbb{R} / \mathbb{Z} \backslash F)\right]=\operatorname{colim}_{F \in \mathcal{F}^{\prime}} \tilde{X}\left[\pi_{0}(\mathbb{R} / \mathbb{Z} \backslash F)\right]
$$

\subsection{Dihedral simplex $\operatorname{Sim}_{\mathrm{dih}}^{n}$}

Let $\operatorname{Sim}_{\text {cyc }}^{n}$ denote, as in [2], the set of points $\left(x_{0}, \ldots, x_{n}\right) \in(\mathbb{R} / \mathbb{Z})^{n+1}$ such that $x_{0}, \ldots, x_{n}$ are in the correct cyclic order. We also denote by $\operatorname{Sim}_{\text {cyc }}^{n, \text { op }}$ the set of points $\left(x_{0}, \ldots, x_{n}\right) \in(\mathbb{R} / \mathbb{Z})^{n+1}$ such that $x_{n}, \ldots, x_{0}$ are in the correct cyclic order. The geometric realization of the standard dihedral set $\Lambda_{\text {dih }}[n][\cdot]=\operatorname{Hom}_{\Delta D}\left([\cdot],[n]_{\text {cyc }}\right)$ is identified with the disjoint union $\operatorname{Sim}_{\text {dih }}^{n}$ of $\operatorname{Sim}_{\text {cyc }}^{n}$ and $\operatorname{Sim}_{\text {cyc }}^{n \text {,op }}$. This identification is induced from the maps $\phi: \operatorname{Hom}_{\Delta_{\mathrm{big}} D}\left(\pi_{0}(\mathbb{R} / \mathbb{Z} \backslash F),[n]_{\mathrm{cyc}}\right) \rightarrow \operatorname{Sim}_{\mathrm{dih}}^{n}, F \in \mathcal{F}^{\prime}$, given as follows. Let $f: \pi_{0}(\mathbb{R} / \mathbb{Z} \backslash F) \rightarrow[n]_{\text {cyc }}$ be a covariant $\mathbb{Z}_{+}$-functor. We associate to $f$ a point $\phi(f)=\left(x_{0}, \ldots, x_{n}\right) \in \operatorname{Sim}_{\text {cyc }}^{n}$ in the following way. Let $i$ be the smallest integer between 0 and $n$ such that $i /(n+1) \in[n]_{\text {cyc }} \subset \mathbb{R} / \mathbb{Z}$ is in the image of $f$. Suppose that an arc starting from $x \in F$ is sent to $i /(n+1) \in[n]_{\text {cyc }}$, and the preceding arc is not (remember that the arcs are oriented in the cyclic order). We set $x_{0}=x_{1}=\cdots=x_{i}=x$. Next, suppose $j$ be the second smallest in the image of $f$, and that an arc starting from $y \in F$ is sent to $j /(n+1) \in[n]_{\mathrm{cyc}}$ (and the preceding arc is not). We set $x_{i+1}=\cdots=x_{j}=y$. In this way we obtain $x_{0}$ through $x_{k}$, where $k$ 
is the largest in the image of $f$. We finally set $x_{k+1}=\cdots=x_{n+1}=x_{0}$, and thus get $\left(x_{0}, \ldots, x_{n}\right) \in \operatorname{Sim}_{\text {cyc }}^{n}$.

Similarly, we associate to a contravariant $\mathbb{Z}_{+}$-functor $f: \pi_{0}(\mathbb{R} / \mathbb{Z} \backslash F) \rightarrow[n]_{\text {cyc }}$ a point $\phi(f)=\left(x_{0}, \ldots, x_{n}\right) \in \operatorname{Sim}_{\text {cyc }}^{n \text {,op }}$ as follows. Let $i$ be the smallest integer between 0 and $n$ such that $i /(n+1) \in[n]_{\text {cyc }} \subset \mathbb{R} / \mathbb{Z}$ is in the image of $f$. If an arc ending at $x \in F$ is sent to $i /(n+1) \in[n]_{\text {cyc }}$ and the succeeding arc is not, then we set $x_{0}=\cdots=x_{i}=x$. We next suppose $j$ be the second smallest in the image of $f$, and that an arc ending at $y \in F$ is sent to $j /(n+1) \in[n]_{\text {cyc }}$ (and the succeeding arc is not). Then $x_{i+1}=\cdots=x_{j}=y$. We thus obtain $x_{0}$ through $x_{k}$, where $k$ is the largest in the image of $f$. Setting $x_{k+1}=\cdots=x_{n+1}=x_{0}$, we get $\left(x_{0}, \ldots, x_{n}\right) \in \operatorname{Sim}_{\mathrm{cyc}}^{n, \text { op }}$.

Since $\operatorname{Sim}_{\text {cyc }}^{n}$ is homeomorphic to the product of $\mathbb{R} / \mathbb{Z}$ and the standard simplicial $n$-simplex $\Delta^{n}$ (see [2]), and since $\operatorname{Sim}_{\text {cyc }}^{n \text {,op }}$ is homeomorphic to $\operatorname{Sim}_{\text {cyc }}^{n}$ by the orderreversing map $\chi:\left(x_{0}, \ldots, x_{n}\right) \mapsto\left(x_{n}, \ldots, x_{0}\right)$, we see that the dihedral simplex $\operatorname{Sim}_{\text {dih }}^{n}$ is identified with the product of $\Delta^{n}$ and the disjoint union of two copies of $\mathbb{R} / \mathbb{Z}$.

A homeomorphism $\alpha: \mathbb{R} / \mathbb{Z} \rightarrow \mathbb{R} / \mathbb{Z}$ induces a homeomorphism $\alpha \times \cdots \times \alpha$ on $(\mathbb{R} / \mathbb{Z})^{n+1}$. If $\alpha$ is orientation-preserving, then $\alpha \times \cdots \times \alpha$ restricts to a homeomorphism on $\operatorname{Sim}_{\text {cyc }}^{n}$. In this case we also get a homeomorphism on $\operatorname{Sim}_{\text {cyc }}^{n \text {,op via }}$ conjugation by $\chi$. If $\alpha$ is orientation-reversing, then $\alpha \times \cdots \times \alpha$ defines a homeomorphism between $\operatorname{Sim}_{\text {cyc }}^{n}$ and $\operatorname{Sim}_{\text {cyc }}^{n, \text { op }}$. Hence $\alpha$ gives rise to a homeomorphism $\Phi(\alpha)$ on $\operatorname{Sim}_{\mathrm{dih}}^{n}$ that sends a point $\left(x_{0}, \ldots, x_{n}\right) \in \operatorname{Sim}_{\mathrm{cyc}}^{n}$ or $\operatorname{Sim}_{\mathrm{cyc}}^{n, \text { op }}$ to the point $\left(\alpha\left(x_{0}\right), \ldots, \alpha\left(x_{n}\right)\right) \in \operatorname{Sim}_{\mathrm{cyc}}^{n}$ or $\operatorname{Sim}_{\mathrm{cyc}}^{n, \text { op }}$.

\subsection{The Homeo $\mathbb{R} / \mathbb{Z}$-action}

We are now going to complete the proof of Theorem 1.3, by constructing the desired continuous action of Homeo $\mathbb{R} / \mathbb{Z}$ on the realization of a general dihedral set $X[\cdot]$.

A homeomorphism of $\mathbb{R} / \mathbb{Z}$ gives rise to an isomorphism $\rho_{\alpha}:|X[\cdot]| \rightarrow|X[\cdot]|$ defined by

$$
\rho_{\alpha} \circ \operatorname{in}_{F}=\operatorname{in}_{\alpha(F)} \circ \tilde{X}\left[\alpha_{F}^{-1}\right]
$$

where $\alpha_{F}: \pi_{0}(\mathbb{R} / \mathbb{Z} \backslash F) \rightarrow \pi_{0}(\mathbb{R} / \mathbb{Z} \backslash \alpha(F))$ is the covariant or contravariant $\mathbb{Z}_{+}-$ isomorphism induced by $\alpha$. Thus Homeo $\mathbb{R} / \mathbb{Z}$ acts on $|X[\cdot]|$, and the proof of the continuity is as follows, being analogous to the cyclic case (see [2]). First, we have

$$
|X[\cdot]|=\operatorname{colim}_{\Lambda_{\operatorname{dih}}[n][\cdot] \rightarrow X[\cdot]}\left|\Lambda_{\operatorname{dih}}[n][\cdot]\right|=\operatorname{colim}_{\Lambda_{\text {dih }}[n][\cdot] \rightarrow X[\cdot]} \operatorname{Sim}_{\operatorname{dih}}^{n},
$$

and the action of $\alpha$ on $|X[\cdot]|$ is the same as the one induced from the action $\Phi(\alpha)$ of $\alpha$ on $\operatorname{Sim}_{\mathrm{dih}}^{n}$ described above. Hence the continuity of the action is equivalent to the 
continuity of the map Homeo $\mathbb{R} / \mathbb{Z} \rightarrow \operatorname{Hom}_{\mathcal{K}}\left(\operatorname{Sim}_{\text {dih }}^{n}, \operatorname{Sim}_{\text {dih }}^{n}\right), \alpha \mapsto \Phi(\alpha)$. (Here $\mathcal{K}$ denotes the category of $k$-spaces and the sets of maps are topologized by the standard $k$-space topology.)

In the argument below, we use the distance on $\mathbb{R} / \mathbb{Z}$, given by

$$
|x-y|=\min (|x-y|,|x-0|+|1-y|),
$$

where $x$ and $y \in \mathbb{R} / \mathbb{Z}$ in the left-hand side are represented by the unique elements in $[0,1]$, still denoted by $x$ and $y$ and assumed that $x \leq y$, in the right-hand side. We get an induced distance on the product $(\mathbb{R} / \mathbb{Z})^{n+1}$, its subsets $\operatorname{Sim}_{\text {cyc }}^{n}$ and $\operatorname{Sim}_{\text {cyc }}^{n, \text { op }}$, and hence on their disjoint union $\operatorname{Sim}_{\mathrm{dih}}^{n}$. Remember that the sub-basis of the space $\operatorname{Hom}_{\mathcal{K}}\left(\operatorname{Sim}_{\mathrm{dih}}^{n}, \operatorname{Sim}_{\mathrm{dih}}^{n}\right)$ is given by the subsets

$$
N(h, U)=\left\{f: \operatorname{Sim}_{\mathrm{dih}}^{n} \rightarrow \operatorname{Sim}_{\mathrm{dih}}^{n} \mid f(h(K)) \subset U\right\}
$$

where $h: K \rightarrow \operatorname{Sim}_{\text {dih }}^{n}$ is a continuous map from a compact Hausdorff space $K$ and where $U$ is an open set of $\operatorname{Sim}_{\text {dih }}^{n}$. Hence it suffices to show that $\mu_{n}^{-1} N(h, U)$ is open in Homeo $\mathbb{R} / \mathbb{Z}$. To this end, we fix an arbitrary $\alpha \in \mu_{n}^{-1} N(h, U)$ and will show that there is an open neighbourhood $N(\alpha)$ of $\alpha$ in Homeo $\mathbb{R} / \mathbb{Z}$ such that $N(\alpha) \subset \mu_{n}^{-1} N(h, U)$. Since $U \subset \operatorname{Sim}_{\mathrm{dih}}^{n}$ is open, for every $x \in h(K)$, there exists a positive real number $\varepsilon_{x}$ such that $B_{x}^{\prime \prime}=\left\{y \in \operatorname{Sim}_{\mathrm{dih}}^{n}|| y-\mu_{n}(\alpha)(x) \mid<\varepsilon_{x}\right\}$ is contained in $U$. We take a smaller ball $B_{x}^{\prime}=\left\{y \in \operatorname{Sim}_{\text {dih }}^{n}|| y-\mu_{n}(\alpha)(x) \mid<\varepsilon_{x} / n\right\}$ in $B_{x}^{\prime \prime}$, and put $B_{x}=\mu_{n}(\alpha)^{-1}\left(B_{x}^{\prime}\right) \cap h(K)$. Then $\left\{B_{x}\right\}_{x \in h(K)}$ forms an open cover for $h(K)$. A compactness argument tells us that we can choose finite $x^{(1)}, \ldots, x^{(l)} \in h(K)$ such that $h(K)=\bigcup_{j=1}^{l} B_{x^{(j)}}$. If $\overline{B_{x}}$ denotes the closure of $B_{x}$ in $h(K)$, we also have

$$
h(K)=\bigcup_{j+1}^{l} \overline{B_{x^{(j)}}} .
$$

Note that $\overline{B_{x}}$ is compact (because it is a closed set in a compact set). We let $\iota_{x}: \overline{B_{x}} \rightarrow$ $h(K) \rightarrow \operatorname{Sim}_{\text {dih }}^{n}$ be the inclusion, and consider for every $i=1, \ldots, n$ and $j=1, \ldots, l$, the set

$$
N^{\prime}\left(p_{i} \circ \iota_{x^{(j)}}, p_{i}\left(B_{x^{(j)}}^{\prime}\right)\right)=\left\{\beta \in \operatorname{Homeo} \mathbb{R} / \mathbb{Z} \mid \beta\left(p_{i}\left(\overline{B_{x^{(j)}}}\right)\right) \subset p_{i}\left(B_{x^{(j)}}^{\prime}\right)\right\},
$$

where $p_{i}: \operatorname{Sim}_{\text {dih }}^{n} \rightarrow \mathbb{R} / \mathbb{Z}$ is the projection onto the $i^{\text {th }}$ component. Then $N^{\prime}\left(p_{i}\right.$ 。 $\left.\iota_{x^{(j)}}, p_{i}\left(B_{x^{(j)}}^{\prime}\right)\right)$ is an open set in Homeo $\mathbb{R} / \mathbb{Z}$ containing $\alpha$. We also have

$$
\bigcap_{1 \leq i \leq n, 1 \leq j \leq l} N^{\prime}\left(p_{i} \circ \iota_{x^{(j)}}, p_{i}\left(B_{x^{(j)}}^{\prime}\right)\right) \subset \mu_{n}^{-1}(N(h, U)) .
$$


Indeed, let $\beta$ be in the left-hand side and take $x \in h(K)$ arbitrarily. Then there is some $j$ such that $x=\left(x_{1}, \ldots, x_{n}\right) \in \overline{B_{x^{(j)}}}$. For every $1 \leq i \leq n$, we have

$$
\beta\left(p_{i}(x)\right)=\beta\left(x_{i}\right) \in p_{i}\left(B_{x^{(j)}}^{\prime}\right) \subset\left\{y_{i} \in[0,1]|| y_{i}-p_{i}\left(\mu_{n}(\alpha)\left(x^{(j)}\right)\right) \mid<\varepsilon_{x^{(j)}} / n\right\} .
$$

Hence,

$$
\left|\mu_{n}(\beta)(x)-\mu_{n}(\alpha)\left(x^{(j)}\right)\right|^{2}=\sum_{i+1}^{n}\left|\beta\left(x_{i}\right)-\alpha\left(x_{i}^{(j)}\right)\right|^{2} \leq \sum_{i=1}^{n} \varepsilon_{x^{(j)}}^{2} / n^{2}=\varepsilon_{x^{(j)}}^{2} / n<\varepsilon_{x^{(j)}}^{2} .
$$

Therefore we see $\mu_{n}(\beta)(x) \in B_{x^{(j)}}^{\prime \prime} \subset U$. This implies $\beta \in \mu_{n}^{-1}(N(h, U))$. Thus we take $N(\alpha)=\bigcap_{1 \leq i \leq n, 1 \leq j \leq l} N^{\prime}\left(p_{i} \circ \iota_{x^{(j)}}, p_{i}\left(B_{x^{(j)}}^{\prime}\right)\right)$, obtaining the desired conclusion. The proof of Theorem 1.3 is now complete.

\section{Subdivisions and proofs of Theorems 1.4 and 1.5}

\subsection{Subdivisions of simplicial and cyclic sets}

Let $X[\cdot]$ be a simplicial set. For every positive integer $r$, let $\operatorname{sd}_{r}: \Delta \rightarrow \Delta$ be the functor defined on objects by $\operatorname{sd}_{r}[n]=[r(n+1)-1]$ and on morphisms by

$$
\operatorname{sd}_{r}[f](a(m+1)+b)=a(n+1)+f(b),
$$

where $f:[m] \rightarrow[n], 0 \leq a<r$, and $0 \leq b \leq m$. The $r$-fold edgewise subdivision of $X[\cdot]$ is defined to be the composite $\operatorname{sd}_{r} X[\cdot]=X[\cdot] \circ \mathrm{sd}_{r}$, which is again a simplicial set.

If $X[\cdot]$ is a cyclic set, its subdivisions are defined analogously, but they are not cyclic sets but $\Delta_{r} C$-sets. The category $\Delta_{r} C$, for each $r$, is defined to make the family $\left\{C_{r(n+1)}\right\}_{n \geq 0}$ into a crossed simplicial group, by using the $\mathbb{Z}_{+}$-category $\mathbb{R} / r \mathbb{Z}$ instead of $\mathbb{R} / \mathbb{Z}$. Denote by $[n]_{r}$ the subset (considered as a $\mathbb{Z}_{+}-$subcategory) $\{[(k(n+1)+l) /(n+1)] \mid 0 \leq k<r, 0 \leq l \leq n\}$ of $\mathbb{R} / r \mathbb{Z}$. We define $\Delta_{r} C$ to have as objects the $\mathbb{Z}_{+}$-categories $[n]_{r} \subset \mathbb{R} / r \mathbb{Z}, n \geq 0$, and to have as morphisms from $[m]_{r}$ to $[n]_{r}, \mathbb{Z}_{+}$-functors satisfying $f(x+1)=f(x)+1$. The simplicial index category $\Delta$ is embedded into $\Delta_{r} C$ via the functor that sends $[n]$ to $[n]_{r}$ and $f:[m] \rightarrow[n]$ to $f_{r}:[m]_{r} \ni(k(m+1)+l) /(m+1) \mapsto(k(n+1)+f(l)) /(n+1) \in[n]_{r}$.

Let $\operatorname{sd}_{r}: \Delta_{r} C \rightarrow \Delta C$ be the functor that is defined on objects by

$$
\operatorname{sd}_{r}[n]_{r}=[r(n+1)-1]_{\mathrm{cyc}}
$$

and on morphisms by

$$
\operatorname{sd}_{r}[f]=\rho_{n}^{-1} \circ f \circ \rho_{m}:[r(m+1)-1]_{\mathrm{cyc}} \rightarrow[r(n+1)-1]_{\mathrm{cyc}},
$$


where $f:[m]_{r} \rightarrow[n]_{r}$ is a map in $\Delta_{r} C$ and $\rho_{m}$ and $\rho_{n}$ are the set bijections from $[m]_{r}$ to $[r(m+1)-1]_{\mathrm{cyc}}$, and from $[n]_{r}$ to $[r(n+1)-1]_{\mathrm{cyc}}$, respectively, induced by the isomorphism $\rho: \mathbb{R} / r \mathbb{Z} \rightarrow \mathbb{R} / \mathbb{Z}, x \mapsto x / r$. This functor is an extension of the subdivision functor for simplicial sets, in the sense that the diagram

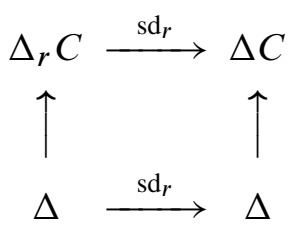

commutes. The $r$-fold edgewise subdivision $\operatorname{sd}_{r} X[\cdot]$ of the cyclic set $X[\cdot]$ is defined to be the composite $X[\cdot] \circ \mathrm{sd}_{r}$.

\subsection{Proof of Theorem 1.4}

Case of simplicial sets By Drinfeld's formula for simplicial sets, we have

$$
\left|\operatorname{sd}_{r} X[\cdot]\right|=\operatorname{colim}_{F \in \mathcal{F}} \widetilde{\operatorname{sd}_{r} X}\left[\pi_{0}([0,1] \backslash F)\right] .
$$

For each $F \in \mathcal{F}$, let $F_{r}$ denote the finite set $\{n+x \mid 0 \leq n<r, x \in F \cup\{0,1\}\} \subset[0, r]$. If $n$ is the cardinality of $\pi_{0}([0,1] \backslash F)$, then that of $\pi_{0}\left([0, r] \backslash F_{r}\right)$ is $r n$. In this case we have $\widetilde{\operatorname{sd}_{r} X}\left[\pi_{0}([0,1] \backslash F)\right]=\operatorname{sd}_{r} X[n-1]=X[r n-1]=\tilde{X}\left[\pi_{0}\left([0, r] \backslash F_{r}\right)\right]$ by construction. Therefore the realization of the subdivision can be rewritten as

$$
\left|\operatorname{sd}_{r} X[\cdot]\right|=\operatorname{colim}_{F \in \mathcal{F}} \tilde{X}\left[\pi_{0}\left([0, r] \backslash F_{r}\right)\right] .
$$

Now we compare the index categories of the colimits $\operatorname{colim}_{F \in \mathcal{F}} \tilde{X}\left[\pi_{0}\left([0, r] \backslash F_{r}\right)\right]$ and $\operatorname{colim}_{F \in \mathcal{F}_{r}} \tilde{X}\left[\pi_{0}([0, r] \backslash F)\right]$. For every $F \in \mathcal{F}_{r}$ there exists a set $F^{\prime} \in \mathcal{F}$ such that $F \subset\left(F^{\prime}\right)_{r}$. Indeed, $\{x \in[0,1] \mid n+x \in F$ for some $0 \leq n<r\} \subset[0,1]$ is such a set. This means that the subcategory of $\mathcal{F}_{r}$ consisting of subsets in $[0, r]$ of the form $F_{r}$ with $F \in \mathcal{F}$ is cofinal, whence we obtain the expression of the statement.

Case of cyclic sets In general, it can be likewise proved that the geometric realization of a $\Delta_{r} C$-set $Y[\cdot]$ is given by

$$
\operatorname{colim}_{F} \tilde{Y}\left[\pi_{0}(\mathbb{R} / r \mathbb{Z} \backslash F)\right],
$$

where $F$ runs through finite subsets of $\mathbb{R} / r \mathbb{Z}$ such that card $\pi_{0}(\mathbb{R} / r \mathbb{Z})=r(n+1)$, $n \geq 0$, and where $\widetilde{Y}[\cdot]$ is the extension of $Y[\cdot]$ to the category $\Delta_{r, \operatorname{big}} C$ of $\mathbb{Z}_{+}$ categories isomorphic to some $[n]_{r}$. Thus $\left|\mathrm{sd}_{r} X[\cdot]\right|$ is the colimit

$$
\operatorname{colim}_{F} \widetilde{\operatorname{sd}_{r} X}\left[\pi_{0}(\mathbb{R} / r \mathbb{Z} \backslash F)\right],
$$

which can be deformed into the desired form in a similar way to the previous case. 


\subsection{Subdivisions of dihedral sets and proof of Theorem 1.5}

Define the category $\Delta_{r} D$ to have the same set of objects as $\Delta_{r} C$, and to have as morphisms covariant $\mathbb{Z}_{+}$-functors $f$ satisfying $f(x+1)=f(x)+1$ and contravariant $\mathbb{Z}_{+}$-functors $g$ satisfying $g(x+1)=g(x)-1$. This category contains $\Delta_{r} C$ and, in particular, $\Delta$ as subcategories. We notice that if we write

$$
\tau_{r, n}:[n]_{r} \rightarrow[n]_{r} \quad \omega_{r, n}:[n]_{r} \rightarrow[n]_{r}
$$

for the isomorphisms in $\Delta_{r} D$ given by $\tau_{r, n}(x)=1 /(n+1)+x$ and $\omega_{r, n}(x)=$ $-1 /(n+1)-x$, respectively, for each $n$, then they satisfy the relations

$$
\tau_{r, n}^{r(n+1)}=\omega_{r, n}^{2}=1, \quad \tau_{r, n} \omega_{r, n}=\omega_{r, n} \tau_{r, n}^{-1} .
$$

Moreover, an argument analogous to Proposition 2.1 shows that $\Delta_{r} D$ is generated by $\tau_{r, n}, \omega_{r, n}$, and $f_{r}$ with $f$ in $\Delta$. Therefore $\Delta_{r} D$ makes $\left\{D_{r(n+1)}\right\}_{n \geq 0}$ into a crossed simplicial group. We also note that $\Delta_{r} D$ has a presentation described as follows. If $d^{i}:[n-1] \rightarrow[n]$ and $s^{i}:[n+1] \rightarrow[n], 0 \leq i \leq n$, denote the face and degeneracy operators in $\Delta$, then $\Delta_{r} D$ is generated by $d_{r}^{i}, s_{r}^{i}, \tau_{r, n}$, and $\omega_{r, n}$, subject to the relations:

(S-1) $\quad d_{r}^{i} d_{r}^{j}=d_{r}^{j} d_{r}^{i-1}(j<i)$

(S-2) $s_{r}^{i} s_{r}^{j}=s_{r}^{j-1} s_{r}^{i}(i<j)$

(S-3) $\quad s_{r}^{i} d_{r}^{j}= \begin{cases}d_{r}^{j} s_{r}^{i-1} & (j<i) \\ 1 & (i=j, j-1) \\ d_{r}^{j-1} s_{r}^{i} & (i<j-1)\end{cases}$

(D-1) $\omega_{r, n}^{2}=\tau_{r, n}^{r(n+1)}=1$

(D-2) $\tau_{r, n} \omega_{r, n}=\omega_{r, n} \tau_{r, n}^{-1}$

(SD-1) $\omega_{r, n} d_{r}^{i}=d_{r}^{n-i} \omega_{r, n-1}(0 \leq i \leq n)$

(SD-2) $\omega_{r, n} s_{r}^{i}=s_{r}^{n-i} \omega_{r, n+1}(0 \leq i \leq n)$

(SD-3) $\tau_{r, n} d_{r}^{i}= \begin{cases}d_{r}^{i+1} \tau_{r, n-1} & (i \neq n) \\ d_{r}^{0} & (i=n)\end{cases}$

(SD-4) $\tau_{r, n} s_{r}^{i}= \begin{cases}s_{r}^{i+1} \tau_{r, n+1} & (i \neq n) \\ s_{r}^{0} \tau_{r, n+1}^{2} & (i=n)\end{cases}$

The extended category $\Delta_{r, \text { big }} D$, and the extension of a $\Delta_{r} D$-set $Y[\cdot]$ to

$$
\tilde{Y}[\cdot]:\left(\Delta_{r, \text { big }} D\right)^{\mathrm{op}} \rightarrow \text { Sets }
$$


are defined likewise. The geometric realization of the $\Delta_{r} D$-set $Y[\cdot]$ is given by

$$
\operatorname{colim}_{F} \tilde{Y}\left[\pi_{0}(\mathbb{R} / r \mathbb{Z} \backslash F)\right],
$$

where $F$ runs through finite subsets of $\mathbb{R} / r \mathbb{Z}$ such that card $\pi_{0}(\mathbb{R} / r \mathbb{Z})=r(n+1)$, $n \geq 0$.

Let $X[\cdot]$ be a dihedral set. For each $r$, the dihedral subdivision functor $\operatorname{sd}_{r}: \Delta_{r} D \rightarrow$ $\Delta D$ is constructed in the same way as the cyclic subdivision functor.

Definition 3.1 We define $\operatorname{sd}_{r} X[\cdot]$ to be the $\Delta_{r} D-$ set $X[\cdot] \circ \operatorname{sd}_{\mathrm{r}}:\left(\Delta_{r} D\right)^{\mathrm{op}} \rightarrow$ Sets.

Remark Spaliński [6] defined $\operatorname{sd}_{r} X[\cdot]$ to be the $r$-fold edgewise subdivision of the underlying simplicial set $\left.X\right|_{\Delta^{\text {op }}}[\cdot]$. Our definition is compatible with Spaliński's since we have the following commutative diagram:

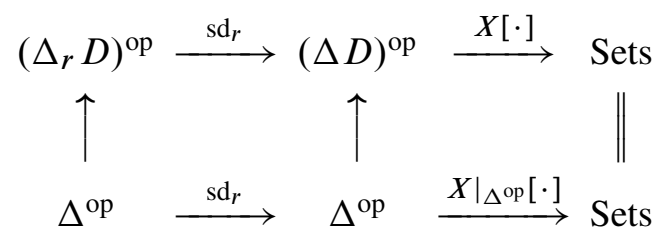

3.3.1 Combination with Quillen and Segal's edgewise subdivision Spaliński [6] introduced another subdivision $\operatorname{sd}_{r}^{\mathrm{e}} X[\cdot]$ of the dihedral set $X[\cdot]$, for each $r \geq 1$, combining $\mathrm{sd}_{r}$ with Quillen and Segal's subdivision functor $\mathrm{sd}^{\mathrm{e}}$ defined in [5]. The functor $\mathrm{sd}^{\mathrm{e}}: \Delta \rightarrow \Delta$ is given on objects by $\mathrm{sd}^{\mathrm{e}}[n]=[2 n+1]$, and on morphisms by $\operatorname{sd}^{\mathrm{e}}[f]=f^{\mathrm{e}}$, where $f:[m] \rightarrow[n]$ is a map in $\Delta$ and $f^{\mathrm{e}}:[2 m+1] \rightarrow[2 n+1]$ is the map defined by $f^{\mathrm{e}}(k)=f(k)$ and $f^{\mathrm{e}}(2 m+1-k)=2 n+1-f(k)$ for $0 \leq k \leq m$. In Spaliński's definition, $\mathrm{sd}_{r}^{\mathrm{e}} X[\cdot]$ is the composite $\left.X\right|_{\Delta^{\mathrm{op}}}[\cdot] \circ \mathrm{sd}_{r} \circ \mathrm{sd}^{\mathrm{e}}$ of the underlying simplicial set of $X[\cdot]$ with $\operatorname{sd}_{r}$ and $\mathrm{sd}^{\mathrm{e}}$.

In fact, $\mathrm{sd}_{r}^{\mathrm{e}} X[\cdot]$ can be defined as a $\Delta_{2 r} D-$ set as follows. Let $\mathrm{sd}_{r}^{\mathrm{e}}: \Delta_{2 r} D \rightarrow \Delta_{r} D$ be the functor that is given on objects by $\operatorname{sd}_{r}^{\mathrm{e}}[n]_{2 r}=[2 n+1]_{r}$ and on morphisms by $\operatorname{sd}_{r}^{\mathrm{e}}\left[\tau_{2 r, n}\right]=\tau_{r, 2 n+1}, \operatorname{sd}_{r}^{\mathrm{e}}\left[\omega_{2 r, n}\right]=\omega_{r, 2 n+1}$, and $\operatorname{sd}_{r}^{\mathrm{e}}\left[f_{2 r}\right]=f_{r}^{\mathrm{e}}$, where $f:[m] \rightarrow[n]$ is a map in $\Delta$ and $f_{r}^{\text {e: }}[2 m+1]_{r} \rightarrow[2 n+1]_{r}$ is the map sending $(2 k(m+1)+l) /(2(m+1))$ to $(2 k(n+1) f(l)) /(2(n+1))$ and $(2 k(m+1)+(2 m+1)-1) /(2(m+1))$ to $(2 k(n+1)+(2 n+1)-f(l) /(2(n+1))$ for $0 \leq k<r, 0 \leq l \leq m$.

Definition 3.2 We define $\operatorname{sd}_{r}^{\mathrm{e}} X[\cdot]$ to be the $\Delta_{2 r} D$-set

$$
X[\cdot] \circ \operatorname{sd}_{r} \circ \operatorname{sd}_{r}^{\mathrm{e}}:\left(\Delta_{2 r} D\right)^{\mathrm{op}} \rightarrow \text { Sets . }
$$


Remark The diagram

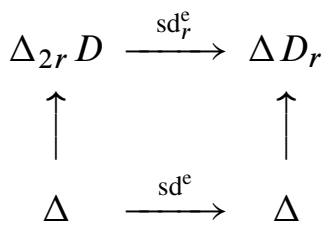

commutes, and in view of this our definition of $\operatorname{sd}_{r}^{\mathrm{e}} X[\cdot]$ is compatible with that of Spaliński [6].

The proof of Theorem 1.5 is similar to Theorem 1.4.

\subsection{Remarks on simplicial actions on subdivisions}

Consider the subgroup of $\operatorname{Aut}_{\Delta_{2 r} D}[n]_{2 r}$ generated by $\tau_{2 r, n}^{2(n+1)}$ and $\omega_{2 r, n}$, which is identified with the dihedral group $D_{r}$. Let $f:[m] \rightarrow[n]$ be a map in $\Delta$ and consider the images

$$
\tau_{r, 2 n+1}^{2(n+1)}, \omega_{r, 2 n+1} \text {, and } f_{r}^{\mathrm{e}} \text { of } \tau_{2 r, n}^{2(n+1)}, \omega_{2 r, n} \text {, and } f_{2 r},
$$

respectively, under the functor $\mathrm{sd}_{r}^{\mathrm{e}}$. Then the diagram

$$
\begin{array}{ccc}
{[2 m+1]_{r} \stackrel{\tau_{r, 2 m+1}^{2(m+1)} \text { or } \omega_{r, 2 m+1}}{\longrightarrow}[2 m+1]_{r}} \\
f_{r}^{\mathrm{e}} \downarrow \\
{[2 n+1]_{r} \stackrel{\tau_{r}^{\mathrm{e}} \downarrow}{\stackrel{\tau_{r, 2 n+1}^{2(n+1)} \text { or } \omega_{r, 2 n+1}}{\longrightarrow}[2 n+1]_{r}}}
\end{array}
$$

commutes. Indeed, for $0 \leq l \leq m$, we have for instance

$$
\begin{aligned}
f_{r}^{\mathrm{e}}\left(\omega_{r, 2 m+1}(l /(2(m+1)))\right) & =f_{r}^{\mathrm{e}}(-1 /(2(m+1))-l /(2(m+1))) \\
& =-1 /(2(n+1))-f(l) /(2(n+1)),
\end{aligned}
$$

and

$$
\begin{aligned}
\omega_{r, 2 n+1}\left(f_{r}^{\mathrm{e}}(l /(2(m+1)))\right. & =\omega_{r, 2 n+1}(f(l) /(2(n+1))) \\
& =-1 /(2(n+1))-f(l) /(2(n+1)) .
\end{aligned}
$$

This means that $D_{r} \subset$ Aut $_{\Delta_{2 r}}[n]_{2 r}$ acts on $\mathrm{sd}_{r}^{\mathrm{e}} X[\cdot]$ simplicially, so that it is possible to define a simplicial set by $[n] \mapsto\left(\operatorname{sd}_{r}^{\mathrm{e}} X[n]\right)^{D_{r}}$. We also note that the action of $D_{r} \subset \operatorname{Aut}_{\Delta_{2 r}}[n]_{2 r}$ on $\left|\mathrm{sd}_{r}^{\mathrm{e}} X[\cdot]\right|$ is nothing but the action obtained by using the action of Homeo $\mathbb{R} / 2 r \mathbb{Z}$ in Theorem 1.5 and by identifying $D_{r}$ with the subgroup of Homeo $\mathbb{R} / 2 r \mathbb{Z}$ generated by $\tau: x \mapsto x+2$ and $\omega: x \mapsto-x$.

We give a new proof to the following result of Spaliński [6]: 
Proposition 3.1 There is a canonical homeomorphism from $\left|\left(\operatorname{sd}_{r}^{\mathrm{e}} X[\cdot]\right)^{D_{r}}\right|$ to $(|X[\cdot]|)^{D_{r}}$.

Proof The left-hand-side is given by the colimit colim $F \in \mathcal{F}\left(\widetilde{\operatorname{sd}_{r}^{\mathrm{e}} X}\left[\pi_{0}([0,1] \backslash F)\right]\right)^{D_{r}}$. If $x \in\left|\operatorname{sd}_{r}^{\mathrm{e}} X[\cdot]\right|=\operatorname{colim}_{F \in \mathcal{F}} \widetilde{\mathrm{sd}_{r}^{\mathrm{e}} X}\left[\pi_{0}([0,1] \backslash F)\right]$ is represented by an element of $\left(\widetilde{\operatorname{sd}_{r}^{\mathrm{e}} X}\left[\pi_{0}([0,1] \backslash F)\right]\right)^{D_{r}}$ with some $F \in \mathcal{F}$, then $x$ is fixed by the $D_{r}$-action on $\left|\operatorname{sd}_{r}^{\mathrm{e}} X[\cdot]\right|$. The converse also holds. Indeed, suppose $x \in\left|\operatorname{sd}_{r}^{\mathrm{e}} X[\cdot]\right|$ to be represented by $y \in \widetilde{\operatorname{sd}_{r}^{\mathrm{e}} X}\left[\pi_{0}([0,1] \backslash F)\right]$ and to be fixed by the $D_{r}$-action. Then for any $\delta \in D_{r}$, there is a larger subset $G \subset[0,1]$ containing $F$ such that the images of $y$ and $\delta \cdot y$ in $\widetilde{\operatorname{sd}_{r}^{\mathrm{e}} X}\left[\pi_{0}([0,1] \backslash G)\right]$ coincides. Then, $x$ is represented by this common element $z \in \widetilde{\operatorname{sd}_{r}^{\mathrm{e}} X}\left[\pi_{0}([0,1] \backslash G)\right]$, and $z$ is fixed by the action of $D_{r}$, ie, $z \in \widetilde{\operatorname{sd}_{r}^{\mathrm{e}} X}\left[\pi_{0}([0,1] \backslash\right.$ $G)])^{D_{r}}$. Therefore:

$$
\begin{aligned}
\left|\left(\operatorname{sd}_{r}^{\mathrm{e}} X[\cdot]\right)^{D_{r}}\right| & =\operatorname{colim}_{F \in \mathcal{F}}\left(\widetilde{\operatorname{sd}_{r}^{\mathrm{e}} X}\left[\pi_{0}([0,1] \backslash F)\right]\right)^{D_{r}} \\
& =\left(\operatorname{colim}_{F \in \mathcal{F}} \widetilde{\operatorname{sd}_{r}^{\mathrm{e}} X}\left[\pi_{0}([0,1] \backslash F)\right]\right)^{D_{r}}=\left(\left|\operatorname{sd}_{r}^{\mathrm{e}} X[\cdot]\right|\right)^{D_{r}}
\end{aligned}
$$

Finally, the canonical homeomorphism from $\left|\operatorname{sd}_{r}^{\mathrm{e}} X[\cdot]\right|$ to $|X[\cdot]|$, which preserves the appropriate actions on both sides, concludes the proof.

We can also consider a simplicial action by the cyclic group $\left\langle\tau_{2 r, n}^{2(n+1)}\right\rangle=C_{r} \subset$ Aut $_{\Delta_{2 r}}[n]_{2 r}$ by restricting the action by $D_{r}$. It is proved likewise that the realization of $\left(\operatorname{sd}_{r}^{\mathrm{e}} X[\cdot]\right)^{C_{r}}$ is canonically homeomorphic to $|X[\cdot]|^{C_{r}}$. Moreover, in this case the simplicial set $\left(\operatorname{sd}_{r}^{\mathrm{e}} X[\cdot]\right)^{C_{r}}$ has an extra structure. Indeed, $\tau_{2 r, n}^{2}$ and $\omega_{2 r, n}$ satisfy

$$
\left(\tau_{2 r, n}^{2}\right)^{n+1}=\omega_{2 r, n}^{2}=1 \quad \text { and } \quad \tau_{2 r, n}^{2} \omega_{2 r, n}=\omega_{2 r, n}\left(\tau_{2 r, n}^{2}\right)^{-1}
$$

on $\left(\operatorname{sd}_{r}^{\mathrm{e}} X[n]\right)^{C_{r}}$. Hence, $\left(\operatorname{sd}_{r}^{\mathrm{e}} X[\cdot]\right)^{C_{r}}$ is again a dihedral set.

\section{References}

[1] M Bökstedt, W C Hsiang, I Madsen, The cyclotomic trace and algebraic $K$-theory of spaces, Invent. Math. 111 (1993) 465-539 MR1202133

[2] V Drinfeld, On the notion of geometric realization, Mosc. Math. J. 4 (2004) 619-626, 782 MR2119142

[3] Z Fiedorowicz, J-L Loday, Crossed simplicial groups and their associated homology, Trans. Amer. Math. Soc. 326 (1991) 57-87 MR998125

[4] J Milnor, The geometric realization of a semi-simplicial complex, Ann. of Math. 65 (1957) 357-362 MR0084138 
[5] G Segal, Configuration-spaces and iterated loop-spaces, Invent. Math. 21 (1973) 213221 MR0331377

[6] J Spaliński, Homotopy theory of dihedral and quaternionic sets, Topology 39 (2000) 557-572 MR1746909

Graduate School of Mathematics, Nagoya University

Furocho, Chikusaku, Nagoya 464-8602, Japan

sho.saito@math.nagoya-u.ac.jp

http://www.math.nagoya-u.ac.jp/ m09019h/

Received: 19 August 2012 Revised: 4 December 2012 